\title{
Rapid vascular regrowth in tumors after reversal of VEGF inhibition
}

\author{
Michael R. Mancuso, ${ }^{1}$ Rachel Davis, ${ }^{1}$ Scott M. Norberg, ${ }^{1}$ Shaun O’Brien,, ${ }^{1}$ Barbara Sennino, ${ }^{1}$ \\ Tsutomu Nakahara, ${ }^{1}$ Virginia J. Yao, ${ }^{1}$ Tetsuichiro Inai, ${ }^{1}$ Peter Brooks, ${ }^{2}$ Bruce Freimark, ${ }^{3}$ \\ David R. Shalinsky, ${ }^{4}$ Dana D. Hu-Lowe, ${ }^{4}$ and Donald M. McDonald ${ }^{1}$
}

\author{
${ }^{1}$ Cardiovascular Research Institute, Comprehensive Cancer Center, and Department of Anatomy, UCSF, San Francisco, California, USA. \\ 2Departments of Radiation Oncology and Cell Biology, New York University Cancer Institute, New York, New York, USA. \\ ${ }^{3}$ Cell-Matrix Inc., a subsidiary of CancerVax, Carlsbad, California, USA. \\ ${ }^{4}$ Department of Research Pharmacology, Pfizer Global Research and Development, San Diego, California, USA.
}

\begin{abstract}
Inhibitors of VEGF signaling can block angiogenesis and reduce tumor vascularity, but little is known about the reversibility of these changes after treatment ends. In the present study, regrowth of blood vessels in spontaneous RIP-Tag2 tumors and implanted Lewis lung carcinomas in mice was assessed after inhibition of VEGF receptor signaling by AG-013736 or AG-028262 for 7 days. Both agents caused loss of $50 \%-60 \%$ of tumor vasculature. Empty sleeves of basement membrane were left behind. Pericytes also survived but had less $\alpha$-SMA immunoreactivity. One day after drug withdrawal, endothelial sprouts grew into empty sleeves of basement membrane. Vessel patency and connection to the bloodstream followed close behind. By 7 days, tumors were fully revascularized, and the pericyte phenotype returned to baseline. Importantly, the regrown vasculature regressed as much during a second treatment as it did in the first. Inhibition of MMPs or targeting of type IV collagen cryptic sites by antibody HUIV26 did not eliminate the sleeves or slow revascularization. These results suggest that empty sleeves of basement membrane and accompanying pericytes provide a scaffold for rapid revascularization of tumors after removal of anti-VEGF therapy and highlight their importance as potential targets in cancer therapy.
\end{abstract}

\section{Introduction}

Inhibitors of VEGF are making their way into the clinic as cancer therapeutics (1-3). In addition to preventing the formation of new tumor vessels, VEGF inhibitors eliminate many existing tumor vessels and tend to normalize the phenotype of tumor vessels that survive treatment (4-7). Regression of tumor vessels caused by VEGF blockade results from loss of endothelial cells, but most pericytes and empty sleeves of basement membrane of the affected vessels persist (6).

Despite conspicuous changes in tumor vasculature induced by inhibition of VEGF signaling, little is known about the reversibility of these changes after cessation of anti-VEGF therapy. Current approaches can effectively inhibit VEGF signaling by blocking the ligand or receptors (8-18), but none permanently stops the production of VEGF or irreversibly disables its receptors. Also, although VEGF inhibitors can destroy as much as $80 \%$ of the tumor vasculature (6), tumor vessels can grow back after cessation of treatment. Indeed, capillaries of the thyroid and trachea, which regress after VEGF inhibition, rapidly regrow when treatment with the inhibitor is stopped $(19,20)$. Tumor burden can increase after treatment ends (21), but to our knowledge, there are no reports of the rate or extent of vascular regrowth in tumors after withdrawal of antiVEGF therapy and little information on conditions that influence the revascularization of tumors. Among the factors that could facilitate the regrowth of tumor vessels are the scaffold of vascular basement membrane and pericytes that remain after endothelial

Conflict of interest: D.D. Hu-Lowe and D.R. Shalinsky are employed by Pfizer Global Research and Development and are holders of company stock and stock options. B. Freimark is a former employee of Cell-Matrix Inc.

Citation for this article: J. Clin. Invest. 116:2610-2621 (2006). doi:10.1172/JCI24612. cells regress $(6,22)$. Basement membrane not only provides tracks for regrowing blood vessels, as for regenerating nerves $(23,24)$, but also serves as a storage site for angiogenic growth factors (25).

The present study sought to determine how rapidly and to what extent tumor blood vessels regrow after cessation of anti-VEGF therapy. In particular, we sought to examine the plasticity of tumor vasculature in response to starting and stopping the inhibition of VEGF signaling, with the goal of determining how rapidly blood vessels in tumors can regrow. With the increasing importance of VEGF inhibitors to the treatment of cancer (1-3), knowing the speed of vascular regrowth in tumors after cessation of treatment is of clinical relevance and could influence the use of VEGF inhibitors.

To address these issues, we took advantage of approaches developed to study VEGF-dependent blood vessels in tumors and normal organs $(6,19,20,26)$. In the present study, VEGF-dependent vessels were defined as blood vessels that regress after inhibition of VEGF. VEGF signaling in tumors in RIP-Tag2-transgenic mice and implanted Lewis lung carcinomas was inhibited with AG-013736, a small molecule inhibitor of VEGF receptor tyrosine kinases known to reduce tumor vascularity $(6,18,27)$. AG-028262, a VEGFR-selective inhibitor with subnanomolar potency (28), was used for comparison. Treatment was stopped after 7 days. The short plasma half-lives of AG-013736 and AG-028262 provided rapid washout and return of VEGF signaling within hours after cessation of treatment $(18,28)$. During the first week after the treatment ended, the amount of vascular regrowth was measured, and the functionality of tumor vessels was tested by assessing vessel patency and endothelial cell VEGFR-2 expression. The extent of VEGF dependence of regrown tumor vessels was determined by giving a second round of treatment. We examined the growth of endothelial sprouts into empty sleeves of basement membrane that remained 

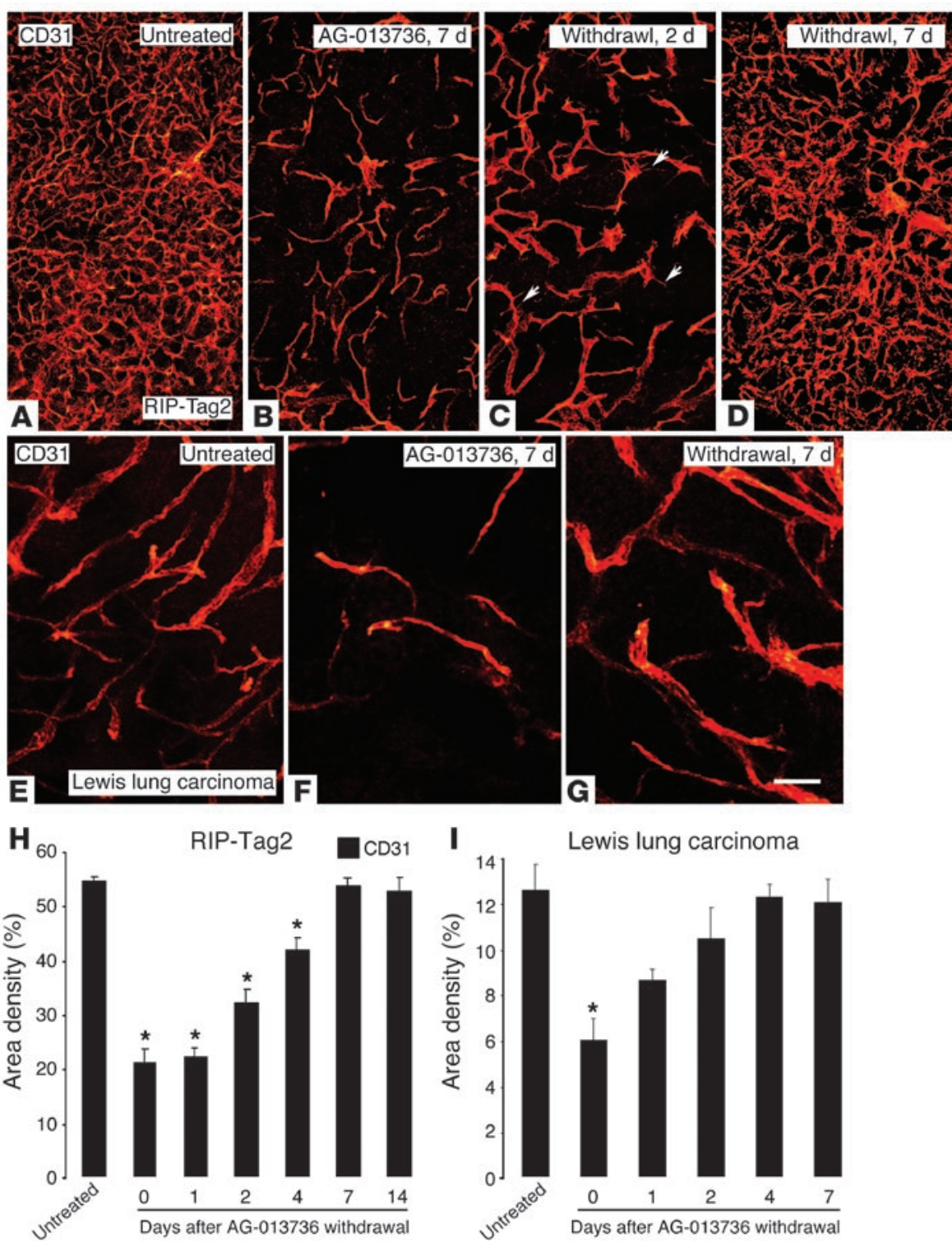

Lewis lung carcinoma

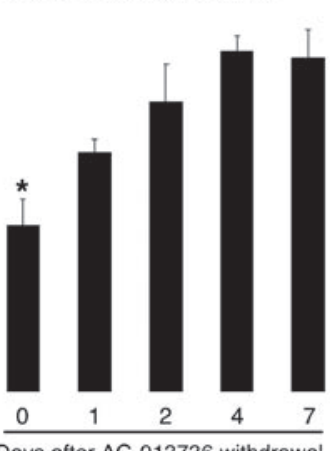

\section{Figure 1}

Regression and regrowth of tumor vessels after VEGF inhibition. Confocal micrographs of RIP-Tag2 tumors compare the dense vascularity of an untreated tumor $(\mathbf{A})$ with the sparse vascularity after AG-013736 treatment for 7 days (B). Tumor vessels that survived treatment had a more uniform caliber and less branching than those in untreated tumors. At 2 days after the treatment ended, vascular sprouts (C, arrows) marked the beginning of vascular regrowth. The projections from vessels were confirmed as sprouts by examining multiple optical sections in the stack of confocal images from the 80- $\mu$ m-thick cryostat section. (D) By 7 days, normalized vessels were replaced by typical tumor vessels. Vascularity of Lewis lung carcinoma $(\mathbf{E})$ was similarly reduced by AG-013736 treatment for 7 days (F). (G) By 7 days after the treatment, vascularity of Lewis lung carcinoma was back to baseline. Bar graphs illustrate changes in area density of CD31-positive vessels in RIP-Tag2 tumors $(\mathbf{H})$ and Lewis lung carcinomas (I) after 7 days of treatment ( 0 days withdrawal) and after the treatment ended. Treatment reduced tumor vascularity in the 2 models by $61 \%$ and $50 \%$, respectively. After AG-013736 treatment was stopped, tumor vascularity was back to baseline by 7 days but from day 7-14 did not increase beyond that present in untreated tumors $(\mathbf{H}) .{ }^{*} P<0.05$ compared with the untreated group. Scale bar (applies to all images): $115 \mu \mathrm{m}$ (A-D); $55 \mu \mathrm{m}$ (E-G). after tumor vessels regressed and compared the rate of regrowth with the rate of disappearance of the sleeves. We also tested whether regrowth was slowed by perturbing the sleeves with an inhibitor of MMPs, AG3340 (29), or a monoclonal antibody (HUIV26) that binds to a cryptic epitope of type IV collagen exposed in basement membrane at sites of vascular remodeling (30-32). Overall, the results indicate that tumors were completely revascularized within the first week after treatment stopped and suggest that surviving pericytes and empty sleeves of vascular basement membrane contributed to the rapid restoration of the tumor vasculature.

\section{Results}

Rapid regrowth of tumor vessels after withdrawal of VEGF inbibition. Blood vessels in untreated RIP-Tag2 tumors were abundant, tortuous, and variable in diameter (Figure 1A). The vascularity of the tumors was conspicuously reduced after treatment with AG-013736 for 7 days (Figure 1B). Tumor vessels that survived the treatment were less tortuous, more uniform in caliber, and had fewer branches and sprouts (Figure 1B). Two days after the treat- ment ended, tumor vessels began to increase in number, became more irregular, and had more sprouts (Figure 1C, arrows). By 7 days after the end of treatment, tumor vessels were as abundant as before the treatment began (Figure 1D). The regrown vessels recapitulated the original tumor vascular architecture.

Blood vessels were less numerous in untreated Lewis lung carcinomas (Figure 1E) than in untreated RIP-Tag2 tumors (Figure 1A), but the response to AG-013736 was similar. Tumor vessels decreased in density sharply during the 7 days of AG-013736 treatment (Figure $1 \mathrm{~F}$ ) and completely regrew within 7 days after the treatment ended (Figure 1G).

In RIP-Tag 2 tumors, overall vascularity decreased by $60 \%$ during 7 days of AG-013736 treatment (Figure 1H). After the inhibitor was stopped, tumor vascularity nearly doubled during the first 4 days and returned to the pretreatment density at day 7 but did not increase further during the second week (Figure 1H). Vascular regrowth was at least as rapid in Lewis lung carcinomas, where the $50 \%$ reduction in vascularity was completely reversed 4 days after the 7-day treatment ended (Figure 1I). 

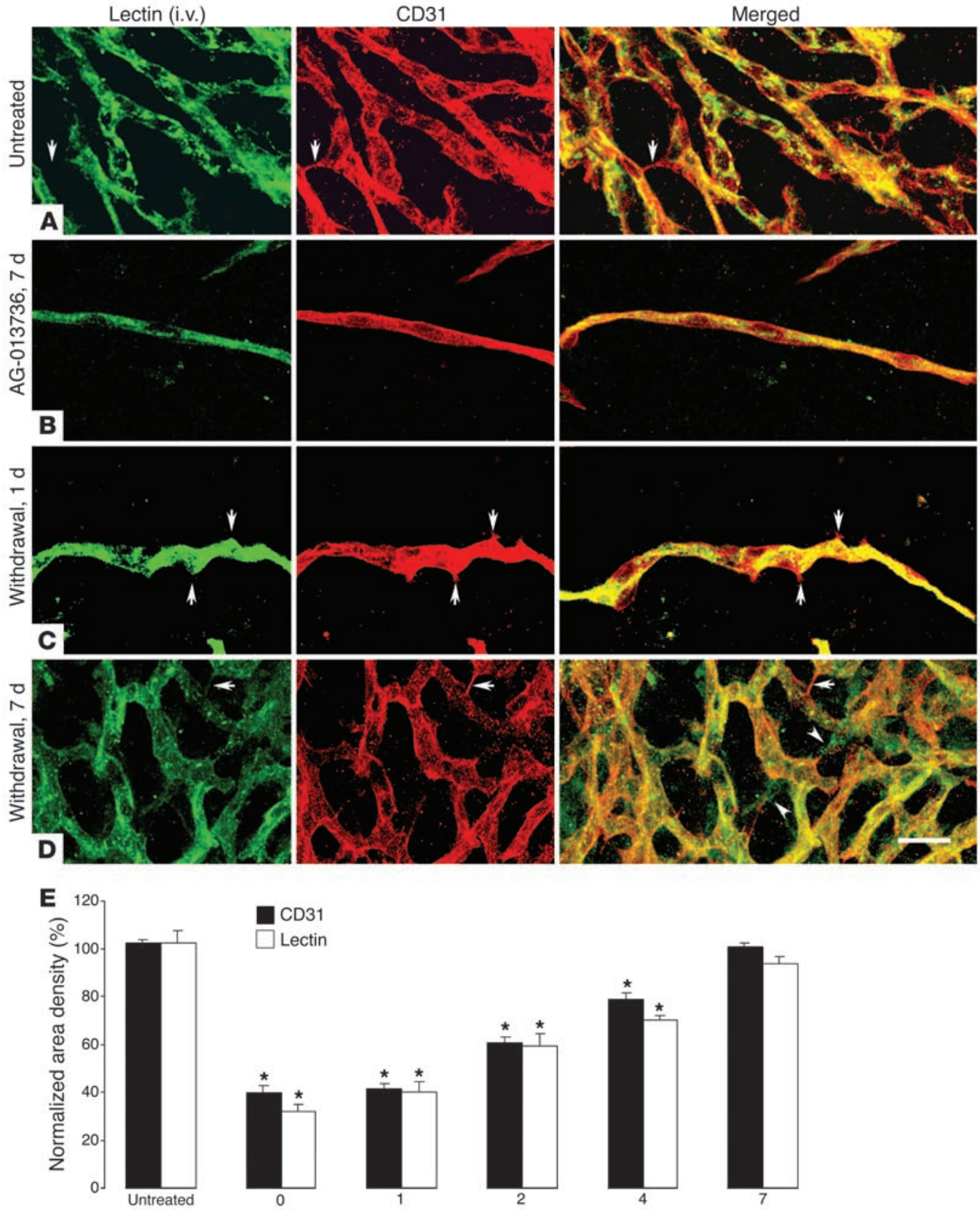

Days after AG-013736 withdrawal

\section{Figure 2}

CD31-positive blood vessels labeled with i.v. FITC-lectin in RIP-Tag2 tumors. Confocal micrographs of tumors: untreated $(\mathbf{A})$; treated with AG-013736 for 7 days (B); and 1 day $(\mathbf{C})$ or 7 days (D) after withdrawal of AG-013736. (A) In the untreated tumor, almost all blood vessels were labeled with lectin, but some CD31positive endothelial sprouts were not labeled (arrows). (B) After treatment, surviving tumor vessels had lectin labeling but lacked sprouts. At 1 day (C) and 7 days (D) after treatment, nearly all tumor vessels were stained with lectin, but lectin-negative endothelial sprouts were abundant (C and D, arrows). Focal regions of extravasated lectin were present at 7 days (D, green, arrowheads in merged image), suggestive of vessel leakiness. (E) Graph showing similarity of area densities of CD31-positive blood vessels and lectinstained vessels during 7 days of vascular regrowth, indicative of vessel functionality. ${ }^{*} P<0.05$ compared with corresponding values for untreated tumors. Scale bar: $22.5 \mu \mathrm{m}$.
Rapid functionality of regrowing tumor vessels. The functionality of regrowing tumor vessels was tested by giving an i.v. injection of fluorescent Lycopersicon esculentum lectin, which binds uniformly and rapidly to the luminal surface of the endothelium and thus labels patent blood vessels, and then staining the tumors for CD31 immunoreactivity $(6,33,34)$. Previous studies using multiple endothelial cell markers verified that essentially all blood vessels in RIP-Tag2 tumors are $\mathrm{CD} 31$ positive $(6,35)$. We also learned that blood vessels undergoing regression lose patency (lectin staining) while remaining CD31 positive $(6,35)$. In the present studies, we found nearly all CD31-positive vessels in untreated RIP-Tag2 tumors labeled with the lectin (Figure $2 \mathrm{~A}$ ). Only endothelial sprouts, which protruded from the walls of some tumor vessels, were lectin negative (Figure 2A, arrows).
After treatment with AG-013736 for 7 days, most surviving vessels in RIP-Tag2 tumors had lectin labeling but did not have sprouts (Figure 2B). One day after the treatment ended, sprouts were the only regions of tumor vessels that lacked lectin labeling (Figure 2C, arrows). By 7 days, lectin stained almost all of the regrown vasculature (Figure $2 \mathrm{D}$, arrows). Measurements confirmed that lectin labeling and vascularity increased in parallel, suggesting that tumor vessels became patent as they regrew to baseline over 7 days (Figure 2E).

Changes in VEGFR-2 immunofluorescence during vascular regression and regrowth. Tumor vessels sensitive to VEGF inhibition have unusually high VEGFR-2 expression (6). Inhibition of VEGF signaling leads to a reduction in VEGFR-2 expression $(6,36,37)$. By 




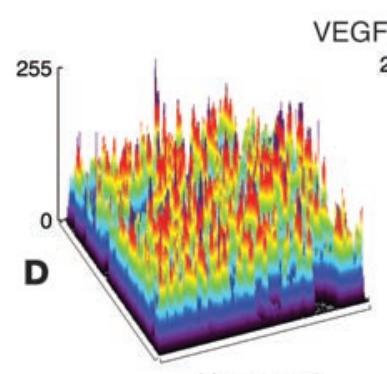

Untreated

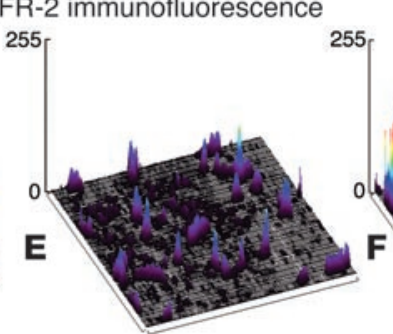

AG-013736, 7 d

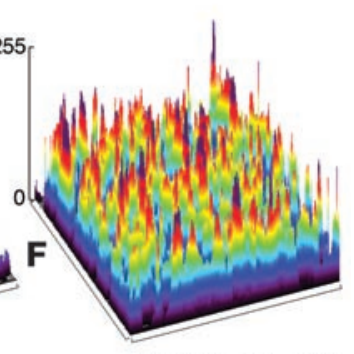

Withdrawal, $7 \mathrm{~d}$

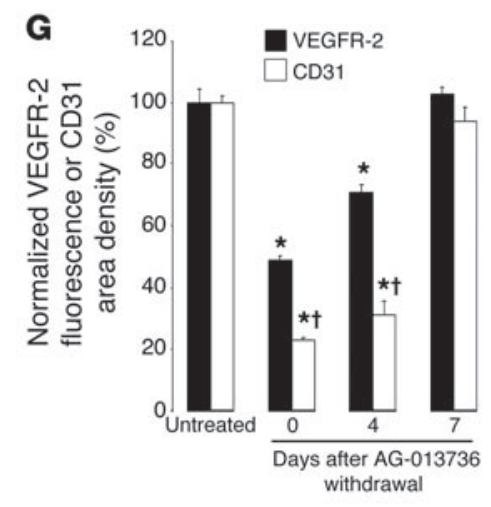

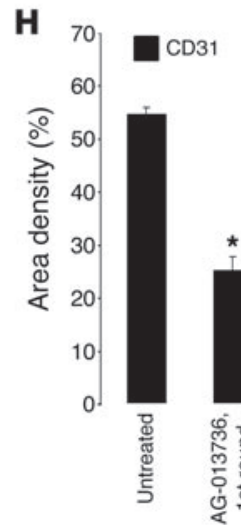

\section{Figure 3}

Reduction in intensity of VEGFR-2 immunofluorescence in RIP-Tag2 tumor vessels after AG-013736 treatment (i.p. injection) for 7 days and during vascular regrowth. Fluorescence micrographs comparing the intense VEGFR-2 immunofluorescence and high vascular density in an untreated tumor $(\mathbf{A})$ with the faint VEGFR-2 immunofluorescence and sparse vasculature in a tumor after 7 days of AG-013736 treatment (B). (C) At 7 days after the treatment ended, both VEGFR-2 immunofluorescence and vascular density returned to baseline. (D-F) Changes in height of peaks in surface plots illustrate the reduction in VEGFR-2 immunofluorescence after 7-day treatment and rebound 7 days thereafter (F). (G) Measurements of VEGFR-2 immunofluorescence show the magnitude of the changes. Comparison of VEGFR-2 immunofluorescence and area density of CD31-positive tumor vessels (both values expressed as percent of corresponding untreated baseline value) suggests that the increase in VEGFR-2 expression preceded the increase in vascular density. (H) Further experiments showed that a second round of AG-013736 reduced tumor vascularity as much as the first round, indicating that much of the regrown tumor vasculature was VEGF dependent. ${ }^{*} P<0.05$ compared with corresponding baseline value. ${ }^{\dagger} P<0.05$, CD31 compared with VEGFR-2. Scale bar: $60 \mu \mathrm{m}(\mathbf{A}-\mathbf{C})$. measuring the fluorescence intensity of RIP-Tag2 tumor vessels stained for VEGFR-2 immunoreactivity, we sought to determine whether the expression of VEGFR-2 in tumor vessels recovered during regrowth and, if so, how quickly. Fluorescent images and surface plots revealed that VEGFR-2 immunoreactivity in untreated RIP-Tag2 tumors (Figure 3, A and D) was conspicuously reduced by treatment with AG-013736 for 7 days (Figure 3, B and E). After drug withdrawal, VEGFR-2 immunofluorescence returned to baseline within 7 days as vascular density increased (Figure 3, C and F). Measurements showed a 52\% reduction in VEGFR-2 immunoreactivity after 7 days of treatment (Figure 3G), consistent with lower VEGFR-2 expression in the surviving vessels. This decrease reversed completely during vascular regrowth and accompanied the return of tumor vascularity to baseline during the week after treatment ended (Figure 3G).

Rapid recovery of VEGF dependence of regrown tumor vessels. The recovery of VEGFR-2 immunoreactivity after withdrawal of VEGF inhibition raised the possibility that regrown vessels in RIP-Tag2 tumors reacquired VEGF dependence - regression after inhibition of VEGF signaling - found in untreated tumors (6). We addressed this question by treating RIP-Tag2 mice with a second round of AG-013736 beginning 7 days after the end of the first round of treat- ment. We found that the second round reduced tumor vascularity as much as the first round (Figure $3 \mathrm{H}$ ). This finding is evidence that much of the regrown tumor vasculature is VEGF dependent.

Changes in pericyte phenotype during vascular regression and regrowth. Treatment with AG-013736 reduces by about one-third the population of $\alpha$-SMA-positive cells in RIP-Tag2 tumors (6). We sought to determine the reversibility of this change and whether it reflects a decrease in number of pericytes or change in pericyte phenotype. Immunohistochemical studies confirmed the decrease in $\alpha$-SMA staining after AG-013736 treatment and revealed that the reduction reversed rapidly after the treatment ended (Figure 4, A-C). Measurements showed that the area density of $\alpha$-SMA, which in these experiments decreased $50 \%$ during treatment, was fully back to baseline by 4 days after the treatment ended (Figure 4D).

To test whether the change in $\alpha$-SMA immunoreactivity reflected a change in pericyte number, we repeated the experiment using a second pericyte marker, PDGFR- $\beta$ (38). Interestingly, PDGFR- $\beta$ immunoreactivity did not undergo a corresponding reduction during treatment and did not change appreciably during regrowth (Figure 4, E-G). To explore further the effects of AG-013736 on $\alpha$-SMA and PDGFR- $\beta$ immunoreactivities, we measured the extent of colocalization of these markers in RIP-Tag2 tumors after treat- 

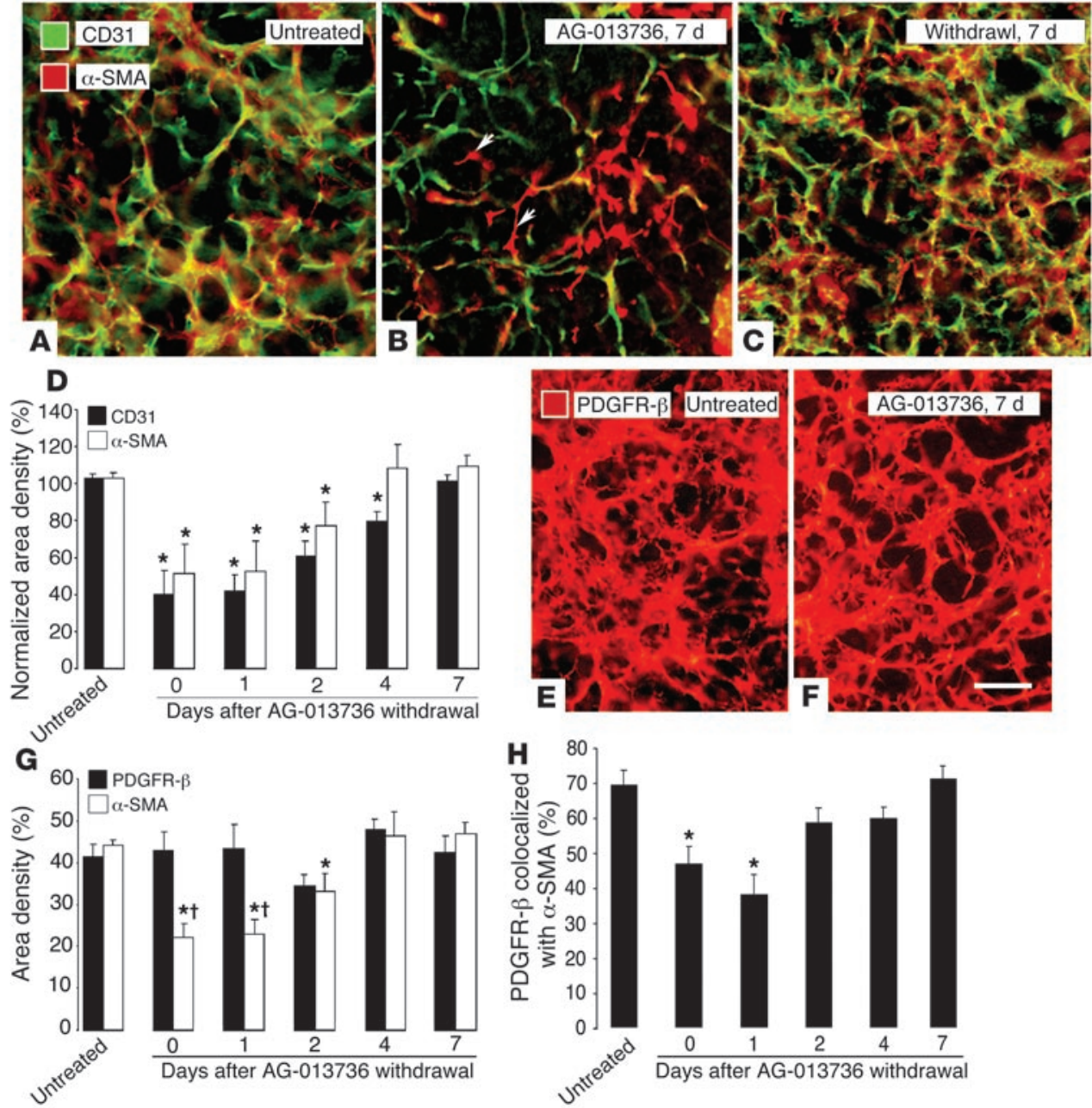

\section{Figure 4}

Changes in pericyte phenotype during regression and regrowth of blood vessels in RIP-Tag2 tumors. Fluorescence microscopic images of tumors stained for CD31 (green, endothelial cells) and $\alpha$-SMA (red, pericytes) comparing untreated vasculature (A), regression after AG-013736 for 7 days (B), and regrowth for 7 days after treatment (C). (D) Area density measurements show that AG-013736 reduced CD31 more than $\alpha$-SMA, but both returned to baseline during 7 days of regrowth. (E and $\mathbf{F}$ ) By comparison, PDGFR- $\beta$ immunoreactivity was not changed by the 7-day treatment. (G) Measurements showed that the area density of PDGFR- $\beta$-positive pericytes, unlike that of $\alpha$-SMA-positive pericytes, remained relatively constant. (G) The ratio of $\alpha$-SMA immunoreactivity to PDGFR- $\beta$ immunoreactivity, illustrated by the amount of colocalization of the 2 markers, decreased after treatment but returned to baseline by 7 days after treatment ended. The stability of the PDGFR- $\beta$-positive pericyte population suggests that the shortterm change in $\alpha$-SMA immunoreactivity reflects a change in pericyte phenotype rather than in pericyte number. ${ }^{*} P<0.05$ compared with control. ${ }^{\dagger} P<0.05$ compared with PDGFR- $\beta$. Scale bar (applies to all images): $85 \mu \mathrm{m}$ (A-C); $100 \mu \mathrm{m}$ (E and F).

ment and during vascular regrowth. The proportion of pixels in which PDGFR- $\beta$ colocalized with $\alpha$-SMA staining that colocalized with $\alpha$-SMA staining - consistent with coexpression in the same cells - decreased during treatment and fully recovered after the treatment ended (Figure $4 \mathrm{H}$ ). Taken together, these results suggest that treatment with AG-013736 induced a reversible change in pericyte phenotype, whereby the reduction in $\alpha$-SMA immunoreactivity reflected a decrease in $\alpha$-SMA protein expression per cell rather than a decrease in cell number.

To determine whether effects of AG-013736 on PDGFR signaling (27) contributed to the change in pericyte phenotype, we exam-



ined for comparison the effects of AG-028262, a potent small molecule inhibitor of VEGFR-2 phosphorylation $\left(\mathrm{IC}_{50}=0.34 \mathrm{nM}\right)$ with little action on PDGFR- $\beta$ $\left(\mathrm{IC}_{50}=193 \mathrm{nM}\right)(28)$. AG-028262 decreased the vascularity of RIPTag2 tumors by $52 \%$ during 7 days of treatment (Figure 5, A, B, and D). After the treatment ended, tumor vessels regrew to baseline within 7 days (Figure 5, C and D). Treatment with AG-028262 did not significantly reduce the number of empty basement membrane sleeves, as reflected by type IV collagen immunoreactivity (Figure 5D). As found with AG-013736, AG-028262 reduced $\alpha$-SMA immunoreactivity (by 54\%) but did not significantly change PDGFR- $\beta$ immunoreactivity (Figure $5 \mathrm{E}$ ). The reduction in $\alpha$-SMA reversed completely over the 7 days after AG-028262 treatment ended (Figure 5E). These results are consistent with the hypothesis that the change in pericyte phenotype after AG-013736 or AG-028262 is a downstream consequence of inhibition of VEGFR signaling rather than a direct effect on PDGF signaling. The regression of endothelial cells left pericytes in empty sleeves of basement membrane (Figure 5, F and G).

Empty sleeves of basement membrane as a scaffold for tumor vessel regrowth. In untreated RIP-Tag2 tumors, CD31 (endothelial cells) and type IV collagen (basement membrane) immunoreactivities had almost identical distributions (Figure 6A). After 7 days of AG-013736 treatment, the overall pattern of basement membrane showed little change, but many endothelial cells regressed, leaving half of the strands of type IV collagen without CD31 immunoreactivity (Figure 6B). By comparison, 7 days after the treatment ended, CD31-positive vessels were as abundant as in control tumors, and few empty sleeves of basement membrane remained (Figure 6C). The amount of type IV collagen immunoreactivity resembled that in the control (Figure 6, A and C). Regrowth of tumor vessels to the original abundance did not increase the overall amount of basement membrane.

The abundance of empty sleeves of basement membrane left behind by regressing tumor vessels and the apparent lack of duplication of vascular basement membrane during regrowth raised the possibility that the sleeves were reused by growing tumor vessels. 

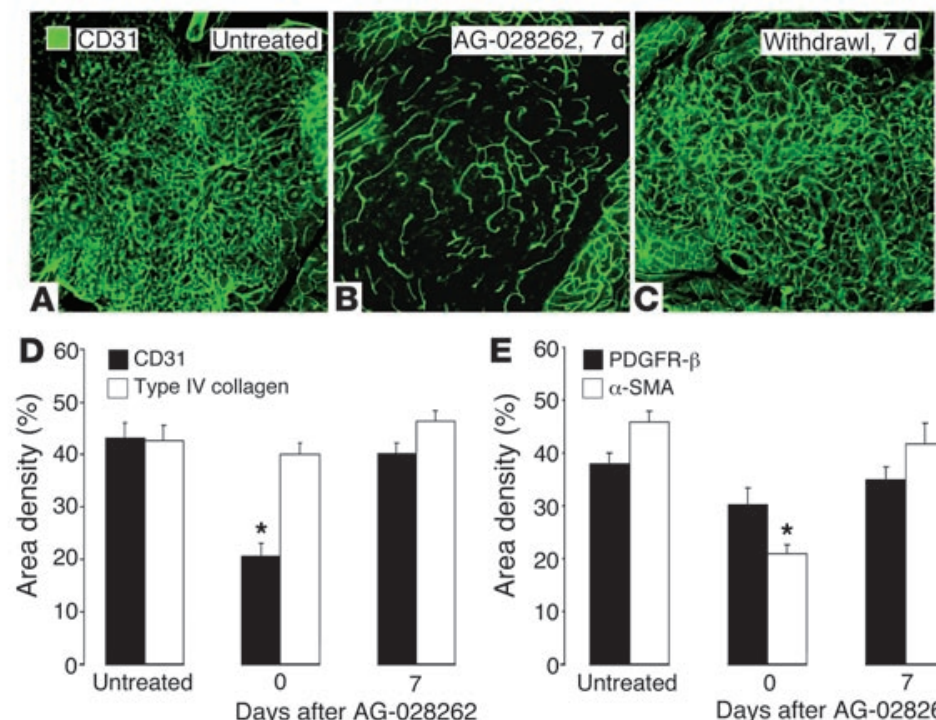

Days after AG-028262 withdrawal
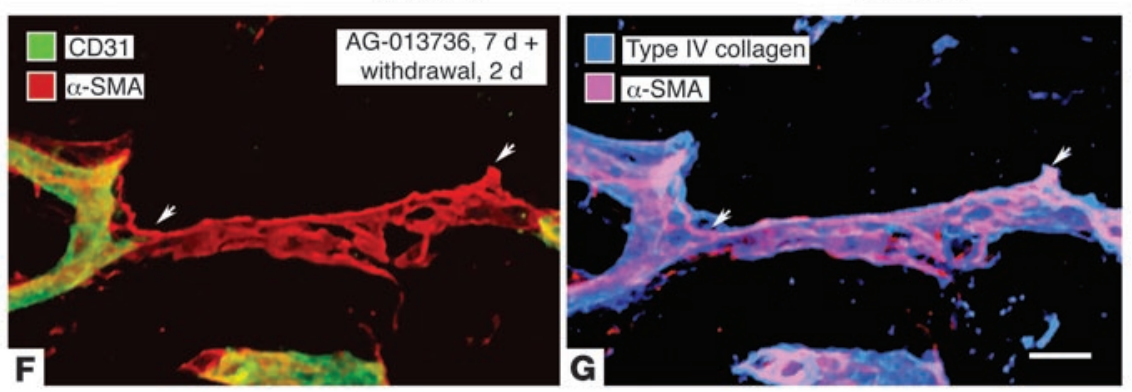

\section{Figure 5}

Selective inhibition of VEGFR signaling by AG-028262 causes reversible changes in endothelial cells and pericytes in RIP-Tag2 tumors. Fluorescence micrographs of tumors stained for CD31 (green) comparing tumor vasculature without treatment $(\mathbf{A})$ with that after AG-028262 treatment for 7 days (B) and 7 days after drug withdrawal (C). (D) Area density measurements show a significant decrease in CD31-positive endothelial cells after AG028262 treatment and complete regrowth after withdrawal for 7 days. (E) $\alpha$-SMA immunoreactivity decreased 54\% during AG-028262 treatment for 7 days ( 0 days withdrawal) but returned to baseline by 7 days after the treatment ended. By comparison, PDGFR- $\beta$ immunoreactivity changed little during treatment or regrowth. (F and $\mathbf{G})$ In RIP-Tag2 tumors treated with AG-013736 followed by 2 days withdrawal, some sleeves of basement membrane contained pericytes but not CD31-positive endothelial cells (region between the arrows). ( $F$ and $\mathbf{G}$ ) Pericytes marked by $\alpha$-SMA immunoreactivity. (G) Basement membrane marked by type IV collagen immunoreactivity. ${ }^{*} P<0.05$ compared with control. Scale bar (applies to all images): $160 \mu \mathrm{m}$ (A-C); $15 \mu \mathrm{m}$ (F and $\mathbf{G}$ ).
Several approaches were used to explore this possibility. We reasoned that if new vessels grew into existing basement membrane sleeves, the rate of vessel regrowth should match the rate of disappearance of empty sleeves. Measurements made at 1, 2, 4, 7, and 14 days after cessation of AG-013736 treatment showed that the events were simultaneous (Figure 6D). At baseline, the area densities of type IV collagen and CD31 were essentially the same, 54.4\% versus $54.7 \%$. After 7 days of AG- 013736 treatment, $50 \%$ of the type IV collagen sleeves were unaccompanied by CD31 (Figure 6D), but these empty sleeves became more sparse during vascular regrowth and were absent by 7-14 days after treatment ended (Figure 6E). Over the same period, the $61 \%$ reduction in tumor vasculature decreased to $0 \%$ (Figure 6E). There was little change in the overall amount of type IV collagen (Figure 6D).

We next looked for endothelial sprouts in empty sleeves of basement membrane during early stages of vascular regrowth. At 1 day, CD31-positive sprouts were abundant in sleeves of type IV collagen (Figure 7, A and B, arrows). Absence of lectin staining indicated that the sprouts lacked a lumen (Figure 7A, arrows). Filopodia emerging from endothelial tip cells in regrowing blood vessels were enveloped by type IV collagen (Figure 7C, arrows).

We also explored strategies to test whether vascular regrowth can be blocked by targeting the empty sleeves of basement membrane. These experiments were designed with the knowledge that the sleeves do not disappear and tumor vessels do not regrow during treatment with AG-013736 for 3 weeks (6). In one approach, we asked whether vascular regrowth was impaired by inhibition of MMPs involved in remodeling of the extracellular matrix (29). We found that treatment with AG-013736 in combination with the MMP inhibitor AG3340 caused a greater amount of vascular regression over 7 days (72\%) than AG-013736 treatment alone (60\%) (Figure 7, D and E). However, continuation of AG3340 treatment for 7 days after the VEGF inhibitor was stopped did not significantly reduce the abundance of empty sleeves of basement membrane or slow revascularization (Figure 7, F and G). Empty sleeves were still present after the combination treatment with AG-013736 and AG3340 (Figure 7E), but they disappeared as tumor vessels regrew despite the continued presence of AG3340 (Figure 7F).

In another approach, we targeted basement membrane sleeves with a monoclonal antibody (HUIV26) directed against a cryptic epitope of type IV collagen that is exposed in angiogenesis (30). The antibody is known to interfere with angiogenesis in several models (30-32). RIP-Tag2 mice received both AG-028262 and HUIV26 for 7 days, and then AG-028262 treatment was stopped and HUIV26 continued for another 7 days. HUIV26 did not augment the loss of tumor vessels or basement membrane sleeves when given with AG-028262 or reduce revascularization when administered alone during the subsequent 7 -day period (Figure $7 \mathrm{H}$ ). The same results were obtained when HUIV26 was given alone for 4 days (data not shown).

Association of VEGF with empty sleeves of basement membrane. Because VEGF binds to basement membrane components (25), we asked whether empty sleeves of basement membrane, formed when tumor vessels regress, are VEGF storage sites that could influence regrowth of tumor vessels. By examining the distribution of VEGF immunoreactivity in RIP-Tag2 tumors treated with AG-013736 for 7 days, we found VEGF concentrated not only in RIP-Tag2 tumor cells (Figure 




Type IV collagen
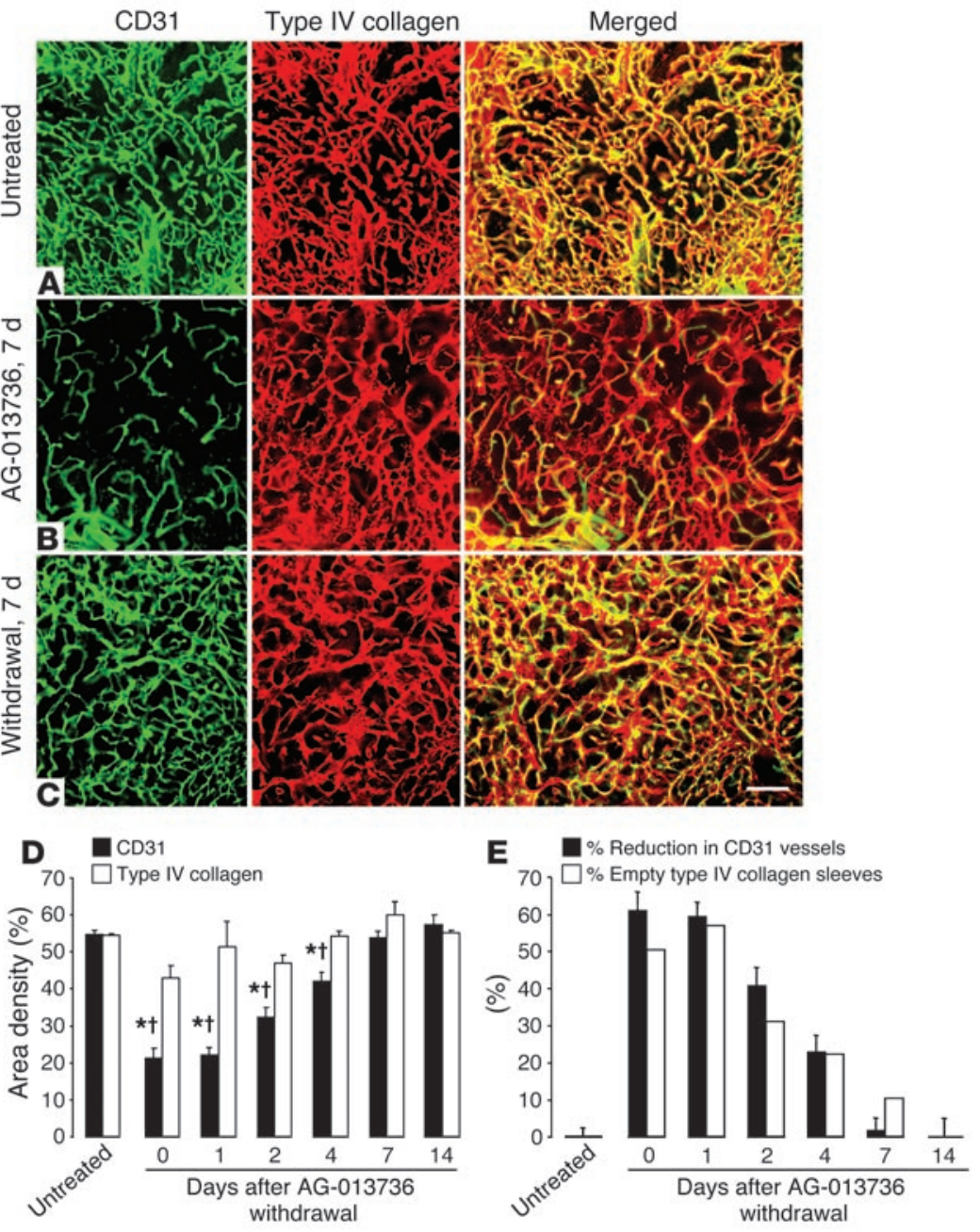

Figure 6

Stability of vascular basement membrane during regression and regrowth of blood vessels in RIP-Tag2 tumors. Fluorescence microscopic images of tumors showing CD31-positive endothelial cells (green), type IV collagen-positive basement membrane (red), and merged images at baseline (A), after AG-013736 treatment for 7 days (B) and after 7-day treatment and 7-day withdrawal (C). The 2 markers colocalized almost completely in untreated RIP-Tag2 tumors (A), but after AG-013736 treatment (B), CD31-positive vessels were sharply reduced but basement membrane was not, as reflected by abundant red strands (B, Merged). (C) By 7 days after treatment ended, tumor vascularity recovered, and most type IV collagen again colocalized with CD31-positive vessels (Merged). (D) Graph of area densities of CD31 and type IV collagen showing that basement membrane remained relatively constant during regression and regrowth of endothelial cells. (E) Graph showing similar rates of tumor vessel regrowth, reflected by CD31 area density, expressed as percent reduction compared with the value for untreated tumors, and disappearance of empty basement membrane sleeves, reflected by the fact that type IV collagen was unaccompanied by CD31, during 14 days after the end of AG-013736 treatment. ${ }^{*} P<0.05$ compared with the untreated group. ${ }^{\dagger} P<0.05$ compared with the corresponding value for type IV collagen. Scale bar: $120 \mu \mathrm{m}(\mathbf{A}-\mathbf{C})$.

7I, arrowheads), as previously reported $(39,40)$, but also in empty sleeves of basement membrane (Figure 7, I and J, arrows).

\section{Discussion}

The goal of this study was to determine the rate and extent of blood vessel regrowth in tumors after removal of VEGF inhibi- tion. The experiments built on previous evidence of robust regression of blood vessels in tumors and certain normal organs after inhibition of VEGF signaling $(6,19,20,26)$. Vascular regrowth in RIPTag2 tumors and Lewis lung carcinomas was evident only 2 days after withdrawal of an inhibitor of VEGF tyrosine kinase receptor signaling and was fully restored by 7 days. In vivo staining with fluorescent lectin showed that new vessels developed a lumen and connection to the circulation as they regrew. Tumor vessels that survived treatment had a uniform caliber, little branching, low VEGFR-2 expression, and independence of VEGF for survival. By comparison, regrowing vessels resembled those in untreated tumors by their tortuosity, high VEGFR-2 expression, and VEGF dependence. Most pericytes survived inhibition of VEGF signaling but had less expression of $\alpha$-SMA. Yet pericyte $\alpha$-SMA expression returned to baseline by 7 days after treatment ended. Empty sleeves of vascular basement membrane appeared as tumor vessels regressed and disappeared as tumor vessels regrew. Inhibition of MMPs or targeting cryptic epitopes of type IV collagen did not eliminate the basement membrane sleeves or prevent rapid revascularization. The results show the reversibility of regression of tumor vessels induced by inhibition of VEGF receptor signaling. Pericytes and empty sleeves of basement membrane may facilitate the regrowth and are thus complementary targets in cancer therapy.

Functional tumor vessels rapidly regrow after removal of VEGF inhibition. VEGF is a key driver of vascular growth in RIP-Tag2 tumors. Tumor vascularization is severely impaired both by genetic inactivation of VEGF alleles in pancreatic $\beta$ cells (40) and by pharmacological inhibition of VEGF signaling (40). The present study examined the regrowth of tumor vessels after reversible inhibition of VEGF receptor signaling by AG- 013736 for 7 days (6), which is not feasible after targeted genetic inactivation of VEGF in RIP-Cre; Vegf $^{\mathrm{f} / \mathrm{fl}}$ mice (40). Loss of tumor vessels and subsequent vascular regrowth were assessed by measuring the abundance of CD31-positive endothelial cells. By comparing CD31 staining with vessel labeling by fluorescent lectin in the bloodstream, we found that most regrowing tumor vessels were patent from day 1 . Only blind-ended tips of sprouts and filopodia lacked a lumen. Similar features have been described in the developing mouse retina, where the lumen of growing blood vessels extends up to endothelial tip cells (41).

Posttreatment restoration of endothelial cell phenotype and VEGF dependence. Reversible changes in VEGFR-2 immunoreactivity reflected the dynamic nature of endothelial cells of tumor vessels during and after treatment. Expression of VEGFR-2 is known to be reduced by inhibition of VEGF signaling $(6,36,37)$. The present study revealed the rapid reversibility of this change. VEGFR-2 immunofluorescence returned to the unusually high intensity typical of RIP-Tag2 tumor vessels by 7 days after cessation of AG-013736 treatment. Recovery 

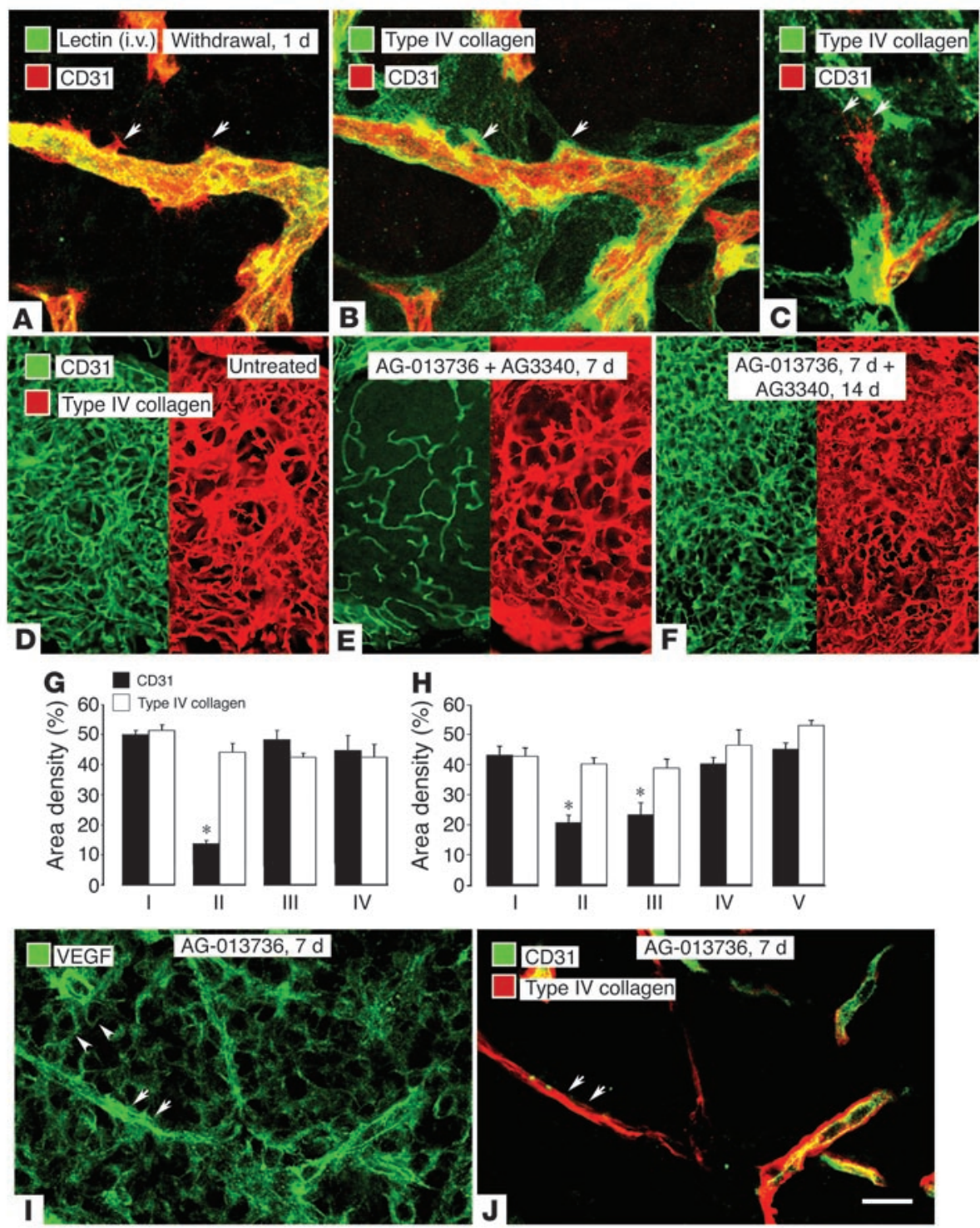

\section{Figure 7}

Vascular basement membrane as a scaffold for regrowth of tumor vessels. (A-C) Vascular regression and regrowth in RIP-Tag2 tumors. (A) Staining with FITClectin and CD31 showed a patent vessel with nonpatent sprouts (arrows) 1 day after AG-013736 treatment ended. (B) Type IV collagen marks loose basement membrane (green). Intense type IV collagen staining next to sprouts (arrows) reflects matrix heterogeneity around regrowing vessel. (C) CD31-positive filopodia (arrows) penetrated basement membrane sleeve. (D-F) Paired images showing that MMP inhibitor AG3340 increased AG-013736-induced vascular regression ( $\mathbf{D}$ and $\mathbf{E}$ ) but did not remove basement membrane sleeves (E) or slow revascularization after AG-013736 treatment ended (F). (G) CD31 and type IV collagen area densities indicated no inhibition of regrowth by MMP inhibition. Groups: (I) vehicle, 7 days; (II) AG-013736 plus AG3340, 7 days; (III) AG-013736 plus AG3340, 7 days, then no treatment, 7 days; (IV) AG-013736 plus AG3340, 7 days, then AG3340 alone, 7 days. (H) Area densities of CD31 and type IV collagen immunoreactivities indicated no inhibition of regrowth by antibody HUIV26. Groups: (I) vehicle, 7 days; (II) AG-028262, 7 days; (III) AG-028262 plus HUIV26, 7 days; (IV) AG-028262, 7 days, then no treatment, 7 days; (V) AG-028262 plus HUIV26, 7 days, then HUIV26 alone, 7 days. (I and J) Sites of VEGF accumulation in basement membrane sleeves (I and $\mathbf{J}$, arrows) and tumor cells (I, arrowheads) in RIP-Tag2 tumor after AG-013736 treatment (i.p. injection) for 7 days. ${ }^{*} P<0.05$ compared with vehicle group. Scale bar (applies to all images): $17 \mu \mathrm{m}$ (A and B); $13 \mu \mathrm{m}$ (C); $160 \mu \mathrm{m}$ (D-F); $20 \mu \mathrm{m}$ (I and J). of high VEGFR-2 expression coincided with the return of VEGF dependence of tumor vessels. In particular, the regrown vessels in RIP-Tag2 tumors were just as sensitive to a second round of VEGF inhibition as was the vasculature under baseline conditions. Studies of another tumor model have shown that the rate of tumor growth can increase after withdrawal of VEGF inhibition (21).

The recovery of VEGF dependence of regrown tumor vessels has potential clinical significance. A brief treatment period was chosen in the present studies of RIP-Tag2 tumors to provide a favorable setting for vascular regrowth after removal of VEGF inhibition to maximize the rate and amount of regrowth. One week of treatment is clearly less than the current clinical use of VEGF inhibitors in treatment of cancer or angiogenic eye diseases, where treatment can last for months or years. Therefore, further experiments are needed to determine whether such prolonged treatment has different effects on regrowth and VEGF dependency of regrowing tumor vessels. In addition, studies with multiple cycles of treatment and withdrawal are needed to learn whether empty basement membrane sleeves eventually disappear and vas- cular regrowth ceases. Indeed, there could be a critical duration of treatment after which regrowth does not occur. In vivo studies and examination of clinical specimens obtained at surgery after one or more courses of VEGF inhibition should make it possible to address these issues in humans.

Changes in pericyte phenotype after VEGF inhibition and during vascular regrowth. Treatment with AG-013736 markedly decreased $\alpha$-SMA immunoreactivity of pericytes in RIP-Tag2 tumors, but PDGFR- $\beta$ immunoreactivity did not show corresponding changes. Because of this difference, the reduction in $\alpha$-SMA immunoreactivity was thought to reflect a change in $\alpha$-SMA expression rather than loss of pericytes. Although AG-013736 inhibits PDGFR- $\beta$, albeit at a concentration 10 times higher than required to inhibit $\operatorname{VEGFR}(18$, $27)$, it is unclear whether the dose of AG-013736 we used would cause sufficient inhibition of PDGFR- $\beta$ in vivo to change pericyte phenotype. To address this issue, we performed complementary studies using AG-028262, which has nearly 1,000-fold greater selectivity for VEGFR than PDGFR (28). Because AG-028262 and AG-013736 had similar effects on pericyte phenotype, the effect on 
$\alpha$-SMA is more likely a downstream consequence of VEGF inhibition than a direct effect on PDGFR- $\beta$.

Signaling mechanisms governing $\alpha$-SMA expression in pericytes are incompletely understood. TGF- $\beta$ upregulates $\alpha$-SMA expression in pericytes (42), and TNF- $\alpha$ decreases $\alpha$-SMA expression (43). Findings in the present studies are consistent with a link between VEGF signaling in endothelial cells and $\alpha$-SMA expression - but not PDGFR- $\beta$ expression. The reduction in $\alpha$-SMA expression may reflect a diminished supply of PDGF from endothelial cells undergoing regression.

Both AG-013736 and AG-028262 were chosen because of their robust, rapid action on blood vessels in RIP-Tag2 tumors and short half-life (approximately 6-7 hours) in the bloodstream $(6,18,28)$. The short half-life made it feasible to study the consequences of inhibition of VEGF signaling followed by rapid reversal after drug withdrawal. We have used macromolecular inhibitors, including soluble decoy receptors and antibodies, in studies of vascular regression in tumors and normal organs $(6,19,20,26)$ but not in the present studies, because their longer half-lives could delay vascular regrowth and lead to underestimate of the rate.

Role of empty sleeves of vascular basement membrane in vascular regrowth. Results of the present study provide clues that empty sleeves of basement membrane facilitate vascular regrowth in tumors. Endothelial cell sprouts were abundant in basement membrane sleeves during the initial phase of regrowth. Although the mere presence of sprouts in the sleeves may be coincidental, the time course of vascular regrowth closely coincided with their disappearance, suggesting that endothelial cells either grew into them or simultaneously created new sleeves as the old ones disappeared. The former possibility seems more likely, because the empty sleeves disappeared over 7-14 days as the vessels regrew but remained for at least 21 days when regrowth was blocked by continuous inhibition of VEGF signaling (6).

Formal proof that basement membrane sleeves are required for rapid regrowth of tumor vessels after regression could be obtained by destroying the sleeves and measuring revascularization. However, we are not aware of an approach that can eliminate the sleeves without causing confounding changes in tumors. One possibility would be to degrade the sleeves with proteases, but proteolytic degradation of basement membrane could liberate proangiogenic and antiangiogenic fragments whose actions would confuse the issue $(44,45)$.

We explored 2 alternatives. In the first, we used AG3340, an inhibitor of MMPs, to perturb remodeling of the extracellular matrix and the release of sequestered VEGF from the matrix (29, $39,46)$. However, we found no reduction in abundance of empty basement membrane sleeves or vascular regrowth. Sleeves were as abundant with the MMP inhibitor as without and were replaced by regrowing vessels, despite the continued presence of the MMP inhibitor. Evidently, MMPs blocked by AG3340 are not essential for formation and persistence of the sleeves or for regrowth of tumor vessels under these conditions.

In another approach, we targeted basement membrane sleeves by using monoclonal antibody HUIV2 6 directed against cryptic domains of denatured type IV collagen (30). In these experiments, RIP-Tag2 mice received AG-028262 in combination with HUIV26 for 7 days and then HUIV26 for another 7 days after AG-028262 treatment was stopped. In these tumors, basement membrane sleeves were present, and blood vessels regrew at the usual rate. It is unclear whether the functional properties of the vessels were the same as in untreated tumors.
Studies of axonal regeneration in peripheral nerves provide clues to the way in which membrane sleeves may facilitate vascular regrowth $(23,24)$. Tubes of Schwann cell basement membrane, left behind when axons degenerate, not only supply chemical guidance signals for axonal regrowth but also serve as an anatomical scaffold for precise functional reinnervation (47). Axons can home to original targets via these tubes with amazing precision (47). Regrowing motor axons can reinnervate their original motor endplates on muscle, provided the tubes are still intact (47). The basement membrane is polarized, and axons grow only along the inner surface (23). Laminins, fibronectin, and heparan sulfate proteoglycan in the basement membrane promote growth. The basement membrane tubes are remarkably durable. They are not degraded by invading macrophages that clear remnants of degenerating axons (48). Segments of denervated nerve continue to be efficient paths for nerve regeneration for several months after denervation (49). Schwann cells, which generally survive nerve transection or crush, play an important role but are not essential for axonal regrowth. Basement membrane tubes can facilitate regrowth into a nerve graft even when all cellular constituents of the graft are eliminated by repeated freeze-thawing to produce an acellular scaffold (23, 48). Acellular grafts, where basement membrane tubes without Schwann cells provide paths for axonal regrowth, can support functional reinnervation over a gap of several centimeters (50) and maintain this potential for months (51).

Tubes of basement membrane in nerves provide cues that determine branching and directional regrowth of axons (47). Empty sleeves of basement membrane may provide similar cues to regrowing blood vessels. Basement membrane is a binding site for heparan sulfate-binding isoforms of VEGF and other factors that can drive vascular regrowth $(25,52,53)$. In RIP-Tag2 tumors, VEGF is stored not only in tumor cells $(39,40)$ but also on empty sleeves of basement membrane. The concentration and distribution of extracellular VEGF shapes the oriented migration and branching of endothelial sprouts (41).

Pericytes contribute to the vascular basement membrane (54). They also play a role in sprouting angiogenesis $(34,35,55)$ and participate in vessel maturation (56-58). Additional studies are needed to determine whether pericytes that survive after endothelial cells regress participate in vascular regrowth in the way Schwann cells facilitate axonal regeneration.

Source of endothelial cells for regrowing tumor vasculature. Under certain conditions, cells from bone marrow contribute to the growth of blood vessels in tumors and other sites of angiogenesis by differentiating into endothelial cells or pericytes or by secreting proangiogenic cytokines $(59,60)$. The mechanism involved is controversial because the magnitude and type of the contribution differs from model to model $(61,62)$. Studies of RIP-Tag2 tumors have shown significant contributions of bone marrow progenitor cells to mural cells of the vasculature and to myofibroblasts in the stroma, but no contribution to the endothelium of tumor vessels has been described $(63,64)$. In the present studies of RIP-Tag 2 tumors, sprouting angiogenesis was extensive during vascular regrowth after removal of VEGF inhibition. Additional experiments are needed to determine whether the new vessels receive a measurable contribution from bone marrow-derived cells.

In conclusion, our findings indicate that tumor blood vessels not only undergo rapid regression when VEGF signaling is inhibited but also equally rapid regrowth after the treatment ends. Regrowing tumor vessels become functional as they form and reacquire 
VEGF as a survival factor. Many pericytes survive VEGF inhibition but undergo a change in phenotype during treatment, reflected by loss of $\alpha$-SMA expression; this change reverses when the treatment stops. Sleeves of basement membrane and accompanying pericytes left behind after endothelial cells degenerate appear to provide a scaffold for regrowth of the tumor microvasculature, analogous to basement membrane tubes and Schwann cells in nerve regeneration. Important next steps will be to examine the impact of longer treatment periods on the rate and extent of vascular regrowth. Overall, these results show that effects of VEGF inhibition on tumors are not permanent and suggest that destroying pericytes and empty sleeves of basement membrane would complement the actions of VEGF inhibitors by decreasing the potential for vascular regrowth.

\section{Methods}

Animals and treatment. Spontaneous pancreatic islet cell carcinomas in RIPTag2 mice (C57BL/ 6 background; ref. 65) from our colony at UCSF were studied at 10-12 weeks of age (6). A $1-\mathrm{mm}^{3}$ piece of Lewis lung carcinoma from a donor mouse was implanted under the dorsal skin of wild-type 7- to 8-week-old C57BL/6 mice for 4 days (6). All experimental procedures were approved by the UCSF Institutional Animal Care and Use Committee.

Drugs. AG-013736, a potent small molecule inhibitor of VEGFR and related receptor tyrosine kinases $(6,18)$, was suspended at a concentration of $5 \mathrm{mg} / \mathrm{ml}$ in $0.5 \%$ carboxymethylcellulose and administered by gavage. In some experiments, AG-013736 was dissolved in 3 parts PEG-400 and 7 parts acidified water ( $\mathrm{pH} 2-3$ ) and injected i.p. In both cases, AG-013736 was administered at a dose of $25 \mathrm{mg} / \mathrm{kg}$ body weight in a volume of $5 \mu \mathrm{l} / \mathrm{g}$, bid. Complementary studies used the selective VEGF receptor tyrosine kinase inhibitor AG-028262 (28), which was prepared at a concentration of $21.92 \mathrm{mg} / \mathrm{ml}$ by suspension in $0.5 \%$ carboxymethylcellulose and administered by gavage ( $40 \mathrm{mg} / \mathrm{kg}$ in a volume of $5 \mu \mathrm{l} / \mathrm{g}$, bid). The effect of metalloproteinase inhibition on regrowth of tumor vessels was assessed by treating mice with AG3340 (29). AG3340, prepared in acidified water, $\mathrm{pH} 2.2-2.3$, was administered by gavage $(200 \mathrm{mg} / \mathrm{kg}$ in a volume of $5 \mu \mathrm{l} / \mathrm{g}$, bid). AG-013736, AG-028262, and AG3340 were supplied by Pfizer Global Research and Development.

The basement membrane of regressing tumor vessels was targeted by treating mice with monoclonal antibody HUIV26, which recognizes cryptic sites on denatured type IV collagen (30-32). HUIV26 at a concentration of $1.15 \mathrm{mg} / \mathrm{ml}$ was injected i.p. at a dose of $100 \mu \mathrm{g}$ (volume, $87 \mu \mathrm{l}$ ) every other day (on days 0, 2, 4, 6 in all mice; days 8 and 10 for 11-day experiments; and days 8,10 , and 12 for 14-day experiments). HUIV2 6 was supplied by Cell Matrix Inc., a subsidiary of CancerVax.

Treatments. Vascular regrowth after AG-013736 treatment was studied in groups of mice that received 1 of 4 treatments: (a) vehicle; (b) AG-013736 for 7 days; (c) AG-013736 for 7 days followed by a period without treatment lasting 1, 2, 4, 7, or 14 days; or (d) AG-013736 for 7 days followed by no treatment for 7 days and then a second 7-day round of AG-013736. Previous studies showed that vehicles for AG-013736 (carboxymethylcellulose or PEG-400 in acidified water) had no detectable effect on RIP-Tag2 tumors (6). AG-028262 was studied in groups similar to a, b, and c. Group c had a 7-day period without treatment.

Effects of AG3340 on regrowth of tumor vessels were studied in groups of mice that received 1 of 4 treatments: (a) vehicle; (b) AG-013736 plus AG3340 for 7 days; (c) AG-013736 plus AG3340 for 7 days, then no treatment for 7 days; or (d) AG-013736 plus AG3340 for 7 days, then AG3340 alone for 7 days.

Effects of HUIV26 were examined in groups of mice that received 1 of 5 treatments: (a) vehicle; (b) AG-028262 for 7 days; (c) AG-028262 plus HUIV26 for 7 days; (d) AG-028262 for 7 days, then no treatment for 4 or 7 days; or (e) AG-028262 plus HUIV26 for 7 days, then HUIV26 alone for 4 or 7 days.
Lectin injection and fixation by vascular perfusion. After the treatment and withdrawal periods, mice were anesthetized with ketamine $(100 \mathrm{mg} / \mathrm{kg}$ i.p.) plus xylazine (10 mg/kg i.p.). Patency of individual tumor vessels was assessed in some mice by injection of $100 \mu \mathrm{l}$ of FITC-labeled L. esculentum lectin ( $1 \mathrm{mg} / \mathrm{ml}$ in $0.9 \% \mathrm{NaCl}$; Vector Laboratories) into a femoral vein 2 minutes before the perfusion. The chest was opened rapidly, and the vasculature was perfused for 2 minutes at a pressure of $120 \mathrm{mmHg}$ with fixative (1\% paraformaldehyde in PBS, $\mathrm{pH} 7.4$; Sigma-Aldrich) from an 18-gauge cannula inserted into the aorta via an incision in the left ventricle. Blood and fixative exited through an opening in the right atrium. Fixed tissues were removed, immersed in fixative for 1 hour at $4{ }^{\circ} \mathrm{C}$, rinsed several times with PBS, infiltrated with $30 \%$ sucrose, frozen in OCT compound, and processed for immunohistochemistry.

Immunohistochemistry. Cryostat sections of tissues were cut at a thickness of $80 \mu \mathrm{m}$ unless otherwise indicated. Sections dried on Superfrost Plus slides (Fisher Scientific) were permeabilized with PBS containing $0.3 \%$ Triton X-100 (LabChem Inc.) and then incubated in a solution containing $5 \%$ normal serum (Jackson ImmunoResearch Laboratories Inc.), PBS-plus (PBS with 0.3\% Triton X-100, 0.2\% BSA; Sigma-Aldrich), and 0.01\% thimerosal (Sigma-Aldrich) for 1 hour at room temperature to block nonspecific antibody binding (6).

Sections on slides were incubated for 12-15 hours with primary antibodies diluted in 5\% normal serum in PBS-plus at room temperature. Endothelial cells, pericytes, and vascular basement membrane of tumor vessels were identified by staining with combinations of 2 or 3 antibodies. Endothelial cells were labeled with rat monoclonal anti-CD31 (PECAM-1, clone MEC 13.3; 1:500; BD Biosciences - Pharmingen) or rabbit polyclonal anti-VEGFR-2 (antibody T014; 1:2,000; gift from R. Brekken and P. Thorpe, University of Texas Southwestern Medical Center, Dallas, Texas, USA). Pericytes were labeled with Cy3-conjugated mouse monoclonal anti- $\alpha$-SMA (clone 1A4; 1:1,000; Sigma-Aldrich) and/or rat monoclonal anti-PDGFR- $\beta$ (clone APB5; 1:2,000; gift from A. Uemura, Kyoto University, Kyoto, Japan). Vascular basement membrane was examined with rabbit polyclonal antitype IV collagen antibody (1:10,000; Cosmo Bio Co.). VEGF was stained with goat polyclonal anti-VEGF antibody (1:400; R\&D Systems).

After incubation with primary antibodies, sections were rinsed with PBS containing $0.3 \%$ Triton X-100 and incubated for 4-6 hours at room temperature with secondary antibodies diluted in $5 \%$ normal serum in PBS-plus. Secondary antibodies were FITC- or Cy3-labeled goat anti-rat IgG for rat anti-CD31 and anti-PDGFR- $\beta$ antibodies; Cy3-, Cy5-, or FITClabeled goat anti-rabbit IgG for rabbit anti-type IV collagen and antiVEGFR-2 antibodies; and Cy3-labeled donkey anti-goat IgG for the goat anti-VEGF antibody (1:400; Jackson ImmunoResearch Laboratories Inc.). Sections were rinsed with PBS containing $0.3 \%$ Triton X-100, postfixed in $4 \%$ paraformaldehyde, rinsed again with PBS, and mounted in VECTASHIELD (Vector Laboratories).

Fluorescence imaging. Tissue sections were examined with a Zeiss Axiophot fluorescence microscope equipped with single, dual, and triple fluorescence filters and a low-light, externally cooled, 3-chip charge-coupled device (CCD) camera (480 × 640-pixel RGB color images; CoolCam; SciMeasure) and with a Zeiss LSM 510 confocal microscope with Argon, Helium-Neon, and UV lasers $(512 \times 512$ - or $1024 \times 1024$-pixel RGB color images; Zeiss).

The anti-CD31 antibody uniformly stained the entire thickness of 80- $\mu \mathrm{m}$ sections of pancreas and tumors of RIP-Tag2 mice. The anti-type IV collagen antibody consistently stained the uppermost $50 \mu \mathrm{m}$ of sections, but staining of deeper regions was variable, perhaps because the dilute antibody $(1: 10,000)$ was depleted during penetration. As a result, CD31positive vessels in the lowermost part of some sections appeared unaccompanied by type IV collagen. This problem was circumvented by obtaining confocal microscopic images from the uppermost $50 \mu \mathrm{m}$ of the sections. 
Tumor vascularity. Endothelial cells, pericytes, and basement membrane of blood vessels in tumors were quantified by measuring the proportion of sectional area (area density) occupied by fluorescence of specific immunohistochemical markers. Tumor vessel patency was assessed by measuring the extent of lectin staining. Digital fluorescence microscopic images, each representing a region measuring $960 \times 1280 \mu \mathrm{m}(\times 10$ objective, $\times 1$ Optovar), were captured from sections of at least 4 RIP-Tag 2 tumors or 2 regions of Lewis lung carcinoma in each mouse. Images were analyzed using ImageJ software (http://rsb.info.nih.gov/ij) (6). Based on an analysis of pixel fluorescence intensities, which ranged from 0 to 255 , specific staining was distinguished from background by empirically using a threshold value of 45 or 50 (6). Area densities of structures stained with lectin, CD31, $\alpha$-SMA, PDGFR- $\beta$, or type IV collagen were calculated as the proportion of pixels having a fluorescence intensity value equal to or greater than the threshold (6). Because the fluorescence threshold was set to provide the greatest signalto-noise ratio for measuring specific immunoreactivity, some of the faintest immunoreactivity may not have been included. However, because thresholds were applied uniformly, all experimental groups were similarly affected.

Intensity of VEGFR-2 immunofluorescence. VEGFR-2 immunofluorescence of RIP-Tag2 tumors was measured in 6 steps (6): (a) Cryostat sections were cut at a thickness of $20 \mu \mathrm{m}$ and stained for VEGFR-2 immunoreactivity or, as a control, with Cy3-labeled secondary antibody without a primary antibody. (b) Camera gain was calibrated on sections stained with the secondary antibody, which by definition had no foreground (specific) fluorescence. Camera gain was set so background fluorescence was barely visible in the digital images ( $\times 20$ objective, $\times 1$ Optovar; tissue region $480 \times 640 \mu \mathrm{m}$ ). Analysis by ImageJ showed that $97 \%$ of pixels had a fluorescence intensity of less than 15 (intensity range, $0-255$ ). The lower limit of specific fluorescence (threshold) was thus established as a fluorescence intensity of 15 . (c) Digital images were then obtained from sections stained for VEGFR-2 immunoreactivity (Cy3-conjugated secondary antibody) with camera gain set as for the calibration sections. Camera gain was adjusted as needed from section to section to maintain the background fluorescence at the calibration level. Brightness of foreground fluorescence was ignored in this step. (d) RGB images were converted to 8-bit grayscale images with ImageJ. (e) Fluorescence intensity was determined for each pixel using ImageJ. (f) Mean fluorescence intensity of these pixels was calculated as the sum of the number of pixels at each intensity of at least 15 , times the intensity, divided by the total number of pixels with intensity of at least 15 .

The mean value for VEGFR-2 fluorescence intensity in RIP-Tag2 tumors in each mouse was calculated from at least 4 images. Group means were expressed as a percentage of the corresponding values for blood vessels in vehicle-treated tumors ( $n=4-6$ mice per treatment group). Tests of reproducibility confirmed that AG-013736 treatment reduced the intensity of VEGFR-2 immunofluorescence of RIP-Tag2 tumors by approximately $50 \%$ in 3 independent experiments.

Colocalization of pericyte markers. As an index of pericyte phenotype, the amount of colocalization of 2 pericyte markers ( $\alpha$-SMA and PDGFR- $\beta$ ) was measured on images of immunohistochemically stained $20-\mu \mathrm{m}$ sections of RIP-Tag2 tumors. Digital images of the red (Cy3-labeled $\alpha$-SMA primary antibody) and green (PDGFR- $\beta$ labeled with FITC-conjugated secondary antibody) channels of the same field were captured separately with the CCD camera on the fluorescence microscope $(\times 10$ objective, $\times 1$ Optovar). The Colocalization plug-in function of ImageJ was used to identify pixels that had a fluorescence intensity equal to or greater than the threshold values (30 to 45 ) in both the red and green channels. The amount of colocalization of the 2 pericyte markers, expressed as the percentage of pixels with abovethreshold PDGFR- $\beta$ immunoreactivity that colocalized with above-threshold $\alpha$-SMA staining, was calculated as the number of colocalized pixels divided by the number of above-threshold pixels in the PDGFR- $\beta$ image.

Statistics. The significance of differences among groups was assessed using ANOVA followed by the Bonferroni-Dunn or Fisher's test for multiple comparisons. Values are expressed as mean $\pm \operatorname{SEM}(n=4-5$ mice per group). $P$ values less than 0.05 were considered significant, except where lower values were indicated in the Bonferroni-Dunn tests.

\section{Acknowledgments}

The authors thank Peter Baluk and Tomomi Kamba for their valuable advice, technical guidance, and suggestions and Douglas Hanahan for supplying breeding pairs for our colony of RIP-Tag2 mice and, with Christopher Chiu, for many insightful and helpful discussions. We thank Jie Wei for genotyping the RIP-Tag2 mice; Rolf Brekken and Philip Thorpe for the VEGFR-2 antibody; Akiyoshi Uemura for the anti-PDGFR- $\beta$ antibody; Pfizer Global Research and Development for the AG-013736, AG-028262, and AG3340; and Cell-Matrix Inc., a subsidiary of CancerVax Corp., for the HUIV26 antibody. This work was supported in part by NIH grants HL-24136 and HL-59157 from the National, Heart, Lung, and Blood Institute and CA082923 and P50-CA90270 from the National Cancer Institute; a grant from Pfizer Global Research and Development San Diego; and funding from AngelWorks Foundation and the Vascular Mapping Project (to D.M. McDonald).

Received for publication January 31, 2005, and accepted in revised form July 18, 2006.

Address correspondence to: Donald M. McDonald, Department of Anatomy, S1363, University of California, 513 Parnassus Avenue, San Francisco, California 94143-0452, USA. Phone: (415) 4762118; Fax (415) 476-4845; E-mail: donald.mcdonald@ucsf.edu.

Tetsuichiro Inai's present address is: Department of Developmental Molecular Anatomy, Graduate School of Medical Sciences, Kyushu University, Fukuoka, Japan.

Bruce Freimark's present address is: Peregrine Pharmaceuticals Inc., Tustin, California, USA.

Rachel Davis, Scott M. Norberg, and Shawn O'Brien contributed equally to this work.
1. Hurwitz, H., et al. 2004. Bevacizumab plus irinotecan, fluorouracil, and leucovorin for metastatic colorectal cancer. N. Engl. J. Med. 350:2335-2342.

2. Wedam, S.B., et al. 2006. Antiangiogenic and antitumor effects of bevacizumab in patients with inflammatory and locally advanced breast cancer. J. Clin. Oncol. 24:769-777.

3. Nieder, C., Wiedenmann, N., Andratschke, N., and Molls, M. 2006. Current status of angiogenesis inhibitors combined with radiation therapy. Cancer Treat Rev. 32:348-364.

4. Jain, R.K. 2001. Normalizing tumor vasculature with anti-angiogenic therapy: a new paradigm for combination therapy. Nat. Med. 7:987-989.

5. Bergers, G., Song, S., Meyer-Morse, N., Bergsland, E., and Hanahan, D. 2003. Benefits of targeting both pericytes and endothelial cells in the tumor vasculature with kinase inhibitors. J. Clin. Invest. 111:1287-1295. doi:10.1172/JCI200317929.

6. Inai, T., et al. 2004. Inhibition of vascular endothelial growth factor (VEGF) signaling in cancer causes loss of endothelial fenestrations, regression of tumor vessels, and appearance of basement membrane ghosts. Am. J. Pathol. 165:35-52.
7. Jain, R.K. 2005. Normalization of tumor vasculature: an emerging concept in antiangiogenic therapy. Science. 307:58-62.

8. Kim, K.J., et al. 1993. Inhibition of vascular endothelial growth factor-induced angiogenesis suppresses tumour growth in vivo. Nature. 362:841-844

9. Witte, L., et al. 1998. Monoclonal antibodies targeting the VEGF receptor-2 (Flk1/KDR) as an antiangiogenic therapeutic strategy. Cancer Metastasis Rev. 17:155-161.

10. Im, S.A., et al. 1999. Antiangiogenesis treatment for 
gliomas: transfer of antisense-vascular endothelial growth factor inhibits tumor growth in vivo. Cancer Res. 59:895-900.

11. Goldman, C.K., et al. 1998. Paracrine expression of a native soluble vascular endothelial growth factor receptor inhibits tumor growth, metastasis, and mortality rate. Proc. Natl. Acad. Sci. U. S. A. 95:8795-8800.

12. Davidoff, A.M., Leary, M.A., Ng, C.Y., and Vanin, E.F. 2001. Gene therapy-mediated expression by tumor cells of the angiogenesis inhibitor flk-1 results in inhibition of neuroblastoma growth in vivo. J. Pediatr. Surg. 36:30-36.

13. Holash, J., et al. 2002. VEGF-Trap: a VEGF blocker with potent antitumor effects. Proc. Natl. Acad. Sci. U. S. A. 99:11393-11398.

14. Millauer, B., Shawver, L.K., Plate, K.H., Risau, W., and Ullrich, A. 1994. Glioblastoma growth inhibited in vivo by a dominant-negative Flk-1 mutant. Nature. 367:576-579.

15. Fong, T.A., et al. 1999. SU5416 is a potent and selective inhibitor of the vascular endothelial growth factor receptor (Flk-1/KDR) that inhibits tyrosine kinase catalysis, tumor vascularization, and growth of multiple tumor types. Cancer Res. 59:99-106.

16. Laird, A.D., et al. 2000 . SU6668 is a potent antiangiogenic and antitumor agent that induces regression of established tumors. Cancer Res. 60:4152-4160.

17. Drevs, J., et al. 2002. PTK787/ZK 222584, a specific vascular endothelial growth factor-receptor tyrosine kinase inhibitor, affects the anatomy of the tumor vascular bed and the functional vascular properties as detected by dynamic enhanced magnetic resonance imaging. Cancer Res. 62:4015-4022.

18. Hu-Lowe, D., et al. 2002. Characterization of potency and activity of the VEGF/PDGF receptor tyrosine kinase inhibitor AG013736. Proc. Am. Assoc. Cancer Res. 43:A5357.

19. Baffert, F., et al. 2006. Cellular changes in normal blood capillaries undergoing regression after inhibition of VEGF signaling. Am. J. Physiol. Heart Circ. Physiol. 290:H547-H559.

20. Kamba, T., et al. 2006. VEGF-dependent plasticity of fenestrated capillaries in the normal adult microvasculature. Am. J. Physiol. Heart Circ. Physiol. 290:H560-H576.

21. Fox, W.D., et al. 2002. Antibody to vascular endothelial growth factor slows growth of an androgen-independent xenograft model of prostate cancer. Clin. Cancer Res. 8:3226-3231.

22. Vracko, R., and Benditt, E.P. 1972. Basal lamina: the scaffold for orderly cell replacement. Observations on regeneration of injured skeletal muscle fibers and capillaries. J. Cell Biol. 55:406-419.

23. Hall, S. 1997. Axonal regeneration through acellular muscle grafts. J. Anat. 190:57-71.

24. Fournier, A.E., and Strittmatter, S.M. 2002. Regenerating nerves follow the road more traveled. Nat. Neurosci. 5:821-822.

25. Kalluri, R. 2003. Basement membranes: structure, assembly and role in tumour angiogenesis. Nat. Rev. Cancer. 3:422-433.

26. Baffert, F., et al. 2004. Age-related changes in vascular endothelial growth factor dependency and angiopoietin-1-induced plasticity of adult blood vessels. Circ. Res. 94:984-992.

27. Wickman, G., et al. 2003. Further characterization of the potent VEGF/PDGF receptor tyrosine kinase inhibitor, AG-013736, in preclinical tumor models for its antiangiogenesis and antitumor activity. Proc. Am. Assoc. Cancer Res. 44:A3780.

28. Zou, H.Y., et al. 2004. AG-028262, a novel selective VEGFR tyrosine kinase antagonist that potently inhibits KDR signaling and angiogenesis in vitro and in vivo. Proc. Am. Assoc. Cancer Res. 45:A2578.

29. Shalinsky, D.R., et al. 1999. Broad antitumor and antiangiogenic activities of AG3340, a potent and selective MMP inhibitor undergoing advanced oncology clinical trials. Ann. N. Y. Acad. Sci. 878:236-270.

30. Xu, J., et al. 2001. Proteolytic exposure of a cryptic site within collagen type IV is required for angiogenesis and tumor growth in vivo. J. Cell Biol. 154:1069-1079.

31. Hangai, M., et al. 2002. Matrix metalloproteinase-9dependent exposure of a cryptic migratory control site in collagen is required before retinal angiogenesis. Am. J. Pathol. 161:1429-1437.

32. Roth, J.M., et al. 2006. Inhibition of experimental metastasis by targeting the HUIV26 cryptic epitope in collagen. Am. J. Pathol. 168:1576-1586.

33. Hashizume, H., et al. 2000. Openings between defective endothelial cells explain tumor vessel leakiness. Am. J. Pathol. 156:1363-1380.

34. Morikawa, S., et al. 2002. Abnormalities in pericytes on blood vessels and endothelial sprouts in tumors. Am. J. Pathol. 160:985-1000.

35. Baluk, P., Morikawa, S., Haskell, A., Mancuso, M., and McDonald, D.M. 2003. Abnormalities of basement membrane on blood vessels and endothelial sprouts in tumors. Am. J. Pathol. 163:1801-1815.

36. Huang, J., et al. 2003. Regression of established tumors and metastases by potent vascular endothelial growth factor blockade. Proc. Natl. Acad. Sci. U. S. A. 100:7785-7790.

37. Zhang, W., Ran, S., Sambade, M., Huang, X., and Thorpe, P.E. 2002. A monoclonal antibody that blocks VEGF binding to VEGFR2 (KDR/Flk-1) inhibits vascular expression of Flk-1 and tumor growth in an orthotopic human breast cancer model. Angiogenesis. 5:35-44.

38. Uemura, A., et al. 2002. Recombinant angiopoietin-1 restores higher-order architecture of growing blood vessels in mice in the absence of mural cells. J. Clin. Invest. 110:1619-1628. doi:10.1172/ JCI200215621.

39. Bergers, G., et al. 2000. Matrix metalloproteinase-9 triggers the angiogenic switch during carcinogenesis. Nat. Cell Biol. 2:737-744.

40. Inoue, M., Hager, J.H., Ferrara, N., Gerber, H.P., and Hanahan, D. 2002. VEGF-A has a critical, nonredundant role in angiogenic switching and pancreatic beta cell carcinogenesis. Cancer Cell. 1:193-202.

41. Gerhardt, H., et al. 2003. VEGF guides angiogenic sprouting utilizing endothelial tip cell filopodia. J. Cell Biol. 161:1163-1177.

42. Verbeek, M.M., Otte-Holler, I., Wesseling, P., Ruiter, D.J., and de Waal, R.M. 1994. Induction of alphasmooth muscle actin expression in cultured human brain pericytes by transforming growth factor-beta 1 . Am. J. Pathol. 144:372-382.

43. Mariani, T.J., Arikan, M.C., and Pierce, R.A. 1999. Fibroblast tropoelastin and alpha-smooth-muscle actin expression are repressed by particulate-activated macrophage-derived tumor necrosis factoralpha in experimental silicosis. Am. J. Respir. Cell Mol. Biol. 21:185-192.

44. Sund, M., Xie, L., and Kalluri, R. 2004. The contribution of vascular basement membranes and extracellular matrix to the mechanics of tumor angiogenesis. APMIS. 112:450-462.

45. Folkman, J. 2004. Endogenous angiogenesis inhibitors. APMIS. 112:496-507.

46. Price, A., et al. 1999. Marked inhibition of tumor growth in a malignant glioma tumor model by a novel synthetic matrix metalloproteinase inhibitor AG3340. Clin. Cancer Res. 5:845-854.
47. Nguyen, Q.T., Sanes, J.R., and Lichtman, J.W. 2002. Pre-existing pathways promote precise projection patterns. Nat. Neurosci. 5:861-867.

48. Ide, C., Tohyama, K., Yokota, R., Nitatori, T., and Onodera, S. 1983. Schwann cell basal lamina and nerve regeneration. Brain Res. 288:61-75.

49. Gulati, A.K. 1996. Peripheral nerve regeneration through short- and long-term degenerated nerve transplants. Brain Res. 742:265-270.

50. Fugleholm, K., Sorensen, J., Schmalbruch, H., and Krarup, C. 1998. Axonal elongation through acellular nerve segments of the cat tibial nerve: importance of the near-nerve environment. Brain Res. 792:309-318.

51. Ochi, M., Wakasa, M., Ikuta, Y., and Kwong, W.H. 1994. Nerve regeneration in predegenerated basal lamina graft: the effect of duration of predegeneration on axonal extension. Exp. Neurol. 128:216-225.

52. Houck, K.A., Leung, D.W., Rowland, A.M., Winer, J., and Ferrara, N. 1992. Dual regulation of vascular endothelial growth factor bioavailability by genetic and proteolytic mechanisms. J. Biol. Chem. 267:26031-26037.

53. Poltorak, Z., et al. 1997. VEGF145, a secreted vascular endothelial growth factor isoform that binds to extracellular matrix. J. Biol. Chem. 272:7151-7158.

54. Jeon, H., et al. 1996. Pericytes from microvessel fragment produce type IV collagen and multiple laminin isoforms. Biosci. Biotechnol. Biochem. 60:856-861.

55. Nehls, V., Denzer, K., and Drenckhahn, D. 1992. Pericyte involvement in capillary sprouting during angiogenesis in situ. Cell Tissue Res. 270:469-474.

56. Beck, L., Jr., and D'Amore, P.A. 1997. Vascular development: cellular and molecular regulation. FASEB J. 11:365-373.

57. Benjamin, L.E., Hemo, I., and Keshet, E. 1998. A plasticity window for blood vessel remodeling is defined by pericyte coverage of the preformed endothelial network and is regulated by PDGF-B and VEGF. Development. 125:1591-1598.

58. Nicosia, R.F., and Villaschi, S. 1995. Rat aortic smooth muscle cells become pericytes during angiogenesis in vitro. Lab. Invest. 73:658-666.

59. Lyden, D., et al. 2001. Impaired recruitment of bone-marrow-derived endothelial and hematopoietic precursor cells blocks tumor angiogenesis and growth. Nat. Med. 7:1194-1201.

60. Kopp, H.G., Ramos, C.A., and Rafii, S. 2006. Contribution of endothelial progenitors and proangiogenic hematopoietic cells to vascularization of tumor and ischemic tissue. Curr. Opin. Hematol. 13:175-181.

61. Li, H., Gerald, W.L., and Benezra, R. 2004. Utilization of bone marrow-derived endothelial cell precursors in spontaneous prostate tumors varies with tumor grade. Cancer Res. 64:6137-6143.

62. Rajantie, I., et al. 2004. Adult bone marrow-derived cells recruited during angiogenesis comprise precursors for periendothelial vascular mural cells. Blood. 104:2084-2086.

63. Casanovas, O., Hicklin, D.J., Bergers, G., and Hanahan, D. 2005. Drug resistance by evasion of antiangiogenic targeting of VEGF signaling in late-stage pancreatic islet tumors. Cancer Cell. 8:299-309.

64. De Palma, M., et al. 2005. Tie2 identifies a hematopoietic lineage of proangiogenic monocytes required for tumor vessel formation and a mesenchymal population of pericyte progenitors. Cancer Cell. 8:211-226.

65. Hanahan, D. 1985. Heritable formation of pancreatic beta-cell tumours in transgenic mice expressing recombinant insulin/simian virus 40 oncogenes. Nature. 315:115-122. 